Proceedings of the 2007 Winter Simulation Conference

S. G. Henderson, B. Biller, M.-H. Hsieh, J. Shortle, J. D. Tew, and R. R. Barton, eds.

\title{
DUALITY IN HIGH LEVEL PETRI-NETS - A BASIS TO DO DIAGNOSES
}

\author{
Jörg R. Müller \\ Eckehard Schnieder \\ Institute for Traffic Safety and Automation Engineering \\ Technical University of Braunschweig \\ 38106 Braunschweig, GERMANY
}

\begin{abstract}
In this paper the relation of high-level Petri-nets (hlpn) and linear algebra is outlined. On the basis of this relation the theory of the dual spaces can be brought in to a new class of hlpn. In this class not only transitions but also places can be marked and each arc is labeled with two mappings, in addition besides transitions also places are firable. By means of an example it is shown that the modified firing rule leads to a behaviour that can be brought in to do diagnoses in hlpn.
\end{abstract}

\section{INTRODUCTION}

The well known relation of (low level) place/transition-nets to linear algebra enables the structural analysis for these nets (see e.g. Murata 1989). Here it is shown that arc-labels in high-level nets (for sake of simplicity we restrict ourselves to predicate/transition-nets (pr/t-nets)) can be interpreted as linear mappings written as tuples. With this relation between pr/t-nets and the theory of linear algebra it is possible even for high-level nets to find applicable structural analysis methods (Müller 2004).

The structure of a (low level) net consists of places, transitions and arcs which represent the flow relation between these two kinds of nodes. If one changes in a (primal) net the direction of each arc and exchanges places with transitions the result is again a net that is called the dual net. This expression isn't new, it has been introduced by Carl Adam Petri (Fernandez 1975). We call the process of creating the dual to a primal net dualizing.

The same duality is achieved, if one transposes the incidence-matrix of a (primal) net and takes the transposed matrix as an incidence-matrix to another net. The latter is the dual to the first.

If one not only dualizes the structure of a net (like Petri) but nets with their markings, a dual behaviour of nets can be achieved, if the tokens are "left lying". As places become transitions, now transitions are marked with tokens. As in a primal net the firing of transitions leads to a changing of the marking of places, in the dual net the firng of places leads to a changing of markings of transitions. A new behaviour in nets (and not only a new interpretation of the dynamic) arises, if we accept tokens on both kinds of nodes. First approaches to apply the duality in place/transition nets can be found in Kruse (2001).

In high-level nets one can distinguish between different tokens. Thus, the arc labels can be formulated in dependence of the existing information. The question arises what there is to do with these arc labels in hlpn. As it is outlined, the arc labels in pr/t-nets can be interpreted as linear mappings written in "tupel-notation". If we presume that the arc labels in $\mathrm{pr} / \mathrm{t}$-nets are of finite dimension they can be represented by matrices of finite dimension. Nets with matrices as arc labels are dualized, by transposing the arc labels (arc matrices), too (Lautenbach 1983). That means: If in a pr/t-net an arc label $\varphi$ - a linear mapping - corresponds to a matrix $M$, in the dual net the arc label again is a linear mapping. Now, this mapping corresponds to the transposed matrix $M^{t}$. Such mappings are referred to as adjoint mappings, they are denoted by $\varphi^{a d}$ : An adjoint mapping $\varphi^{a d}$ is represented by a matrix $M^{t}$ if and only if $\varphi$ is represented by $M$. The basics of duality in hlpn were introduced by Kurt Lautenbach in Lautenbach (1983) with the concept of matrix-nets. In Müller (2004) the duality in hlpn was introduced on the basis of the theory of the dual spaces. In all these approaches the dynamic was defined in a way, that (in low level nets) different kinds of tokens block the firing of nodes and in high level nets tuples block the firing of nodes for certain node-colours. Although there are many arguments to follow that interpretation and apply that firing rule, in this paper we will introduce a different interpretation leading to a different firing rule:

If we presume that a system's state (i.e. the model's marking) is not known exactly the interpretation of tupels as the "states that have to be taken into accout" or the "states that can't be ruled out" seems to be appropriate. Against this backdrop it is clear, that the more tuples there 
are on a node, the less we know about the system's state. In contrary to the approaches mentioned above, here a node can only fire if it's marking corresponds to markings of nodes in its pre- and postset "in a positive way". In order to be able to improve the knowledge about the system's state, the markings on nodes have to be (successively) narrowed down. With the help of adjoint mappings and markings on places as well as on transitions the knowledge about the system's state can be improved by passing information about (locally uncertain) system states through the net and influencing the marking on other nodes.

The paper is organized as follows: The aim of the second chapter is to outline the theory of the dual spaces and to introduce adjoint mappings. In the third chapter the above mentioned class of pr/t-nets is introduced. On the basis of this, the fourth chapter presents how to improve the knowledge about an uncertain system state. In the fifth chapter this concept is demonstrated by means of an example from the automotive area. In chapter six the tasks to be performed in the future are outlined.

\section{PRELIMINARIES}

The theory of the dual spaces and in it's context dual and adjoint mappings play a central role in the diagnosis-method to be presented in the next two chapters. The theoretical basics outlined here are presented in many books dealing with "higher" linear algebra (see e.g. Blyth and Robertson 2002, Curtis 1990, Fischer 2002, Gilbert and Gilbert 1994, Kowalsky 1979, Lipschutz and Lipson 2001).

Definition 1 Let $V, W$ be two vector spaces over the same scalar field $K$. The set of all linear mappings from $V$ to $W$ is denoted by $\mathscr{L}(V, W)$.

Definition 2 Let $V$ be a vector space over the field $K$. The set of all linear mappings of $V$ into $K$ is called the dual space of $V$ and is (usually) denoted by $V^{*}$. Thus

$$
V^{*}:=\mathscr{L}(V, K)
$$

Definition 3 Let $V, W$ be eucledian spaces over the same field $K$; let further be

$$
\begin{aligned}
& \varphi \in \mathscr{L}(V, W) \text { and } \\
& w^{*} \in \mathscr{L}(W, K) \quad\left(=: W^{*}\right),
\end{aligned}
$$

then the dual mapping $\varphi^{*}$ of $\varphi$ is defined as follows:

$$
\begin{aligned}
\varphi^{*} & : \quad \mathscr{L}(W, K) \longrightarrow \mathscr{L}(V, K), \text { with } \\
\varphi^{*}\left(w^{*}\right) & =w^{*} \circ \varphi
\end{aligned}
$$

(with $\circ$ be the composition of linear mappings).
Definition 4 Let $V$ be an eucledian vector space; then the mapping

$$
\begin{aligned}
\Phi & : \quad V \longrightarrow V^{*}, \text { with } \\
\Phi(v) & :=\langle v,\rangle
\end{aligned}
$$

is called a canonical mapping from $V$ to $V^{*}$.

Definition 5 Let $V, W$ be eucledian spaces with inner product '•' over the same field $K$. For each $\varphi \in \mathscr{L}(V, W)$ the adjoint mapping $\varphi^{a d}$ is the mapping

$$
\varphi^{a d} \in \mathscr{L}(W, V)
$$

that is defined by the equation

$$
\varphi(v) \bullet w=v \bullet \varphi^{a d}(w)
$$

for all $v \in V, w \in W$.

Definition 6 Let $V$ be a vector space of dimension $n$ over the field $K$; then the set

$$
B^{n}:=\left\{e_{i} \in K^{n} \mid \forall j \neq i \quad e_{i}(i)=1 \wedge e_{i}(j)=0\right\}
$$

is called the standard basis of $V$.

Theorem 1 If the vector spaces $V$ and $W$ are of finite dimension, then to every $\varphi \in \mathscr{L}(V, W)$ the adjoint $\varphi^{\text {ad }}$ exists (and vice versa).

Let $\left\{e_{1}, e_{2}, \ldots, e_{n}\right\}$ be the standard basis of $V$, then

$$
\varphi^{a d}(w)=\sum_{i=1}^{n}\left(w \bullet \varphi\left(e_{i}\right)\right) \cdot e_{i}
$$

holds.

Proof See e.g. Kowalsky (1979).

We will often write a set of values $\left\{a_{1}, \ldots, a_{n}\right.$ as $\sum_{i=1}^{n} a_{i}$. That means in the definition above $\varphi^{\text {ad }}$ isasetofvalues.

Corollary 2 Let $\varphi \in \mathscr{L}(V, W)$ be a linear mapping and $\varphi^{\text {ad }} \in \mathscr{L}(W, V)$ it's adjoint mapping. Let further be $\Phi$ and $\Psi$ be canonical mappings, then the diagram in Figure 1 is commutativ in the sense of

$$
\varphi^{a d}=\Phi^{-1} \circ \varphi^{*} \circ \Psi
$$

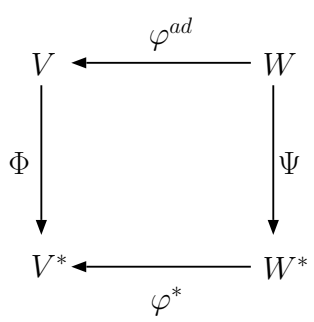

Figure 1: Relation between dual and adjoint mappings. 


\section{DUALITY IN PREDICATE TRANSITION NETS}

The first high-level nets - "high-level" in the sense of nets with individual tokens as markings - were established by Genrich and Lautenbach in 1979 (see Genrich and Lautenbach 1979). Based on Lautenbach and Pagnoni (1984) and Lautenbach and Pagnoni (1985) in Müller (2004) the duality in high-level nets is examined. Each arc of the nets introduced here are labeled with a linear mapping als well as it's adjoint mapping.

Before starting with the definition of (predicate/transition) nets, some technical definitions are to be made:

Definition $7 \quad$ Let $S$ be a set, a multiset $\mathbf{M S}(S)$ over $S$ is defined as a mapping

$$
\text { MS }: S \longrightarrow \mathbb{R}
$$

A multiset is often written as a sum:

$$
\mathbf{M S}(S) \quad:=\sum_{s \in S} \mathbf{M S}(s) \cdot s
$$

Definition 8 The set of all multisets over a set $S$ is denoted as $\mathbb{M S}(S)$.

Definition 9 Let $S$ be a set, then \langle\rangle$_{S}$ is defined as follows:

\langle\rangle$_{S} \quad: \quad S \longrightarrow B^{|S|}-$ and, as an abbreviation:

$\langle S\rangle_{S}:=\left\{e_{i}|i=1, \ldots| S \mid,\right\}$.

In the following we assume that \langle\rangle$_{S}$ is bijective; if $S$ is clear, then we simply write \langle\rangle instead of \langle\rangle$_{S}$ ).

Let $V, W$ be vetor spaces over the same field $K$ and $S_{V}, S_{W}$ be sets with $\left|S_{V}\right|=\operatorname{dim}(V)$ and $\left|S_{W}\right|=\operatorname{dim}(W)$. Then every linear mapping $\varphi \in \mathscr{L}(V, W)$ can be written as

$$
\begin{array}{lll}
\varphi & : \mathbb{M S}\left(\left\langle S_{V}\right\rangle\right) \longrightarrow \mathbb{M} \mathbb{S}\left(\left\langle S_{W}\right\rangle\right), \text { so } \\
\varphi & \in \mathscr{L}\left(\mathbb{M} \mathbb{S}\left(\left\langle S_{V}\right\rangle\right), \mathbb{M S}\left(\left\langle S_{W}\right\rangle\right)\right) .
\end{array}
$$

For example, let $S_{1}$ and $S_{2}$ be given as follows:

$$
\begin{aligned}
& S_{1}=\{(a, a),(a, b),(b, a),(b, b)\} \text { and } \\
& S_{2}=\{a, b\} .
\end{aligned}
$$

Let $\left\langle S_{1}\right\rangle$ and $\left\langle S_{2}\right\rangle$ be given as

$$
\left\langle S_{1}\right\rangle=\left\{\left(\begin{array}{l}
1 \\
0 \\
0 \\
0
\end{array}\right),\left(\begin{array}{l}
0 \\
1 \\
0 \\
0
\end{array}\right),\left(\begin{array}{l}
0 \\
0 \\
1 \\
0
\end{array}\right),\left(\begin{array}{l}
0 \\
0 \\
0 \\
1
\end{array}\right)\right\} \quad \text { with }
$$

$$
\begin{gathered}
(a, a) \longrightarrow\left(\begin{array}{l}
1 \\
0 \\
0 \\
0
\end{array}\right), \\
(a, b) \longrightarrow\left(\begin{array}{l}
0 \\
1 \\
0 \\
0
\end{array}\right), \\
(b, a) \longrightarrow\left(\begin{array}{l}
0 \\
0 \\
1 \\
0
\end{array}\right), \\
(b, b) \longrightarrow\left(\begin{array}{l}
0 \\
0 \\
0 \\
1
\end{array}\right)
\end{gathered}
$$

and $\left\langle S_{2}\right\rangle$ respectively:

$$
\begin{aligned}
\left\langle S_{2}\right\rangle & =\left\{\left(\begin{array}{l}
1 \\
0
\end{array}\right),\left(\begin{array}{l}
0 \\
1
\end{array}\right)\right\} \text { with } \\
a & \longrightarrow\left(\begin{array}{l}
1 \\
0
\end{array}\right), \\
b & \longrightarrow\left(\begin{array}{l}
0 \\
1
\end{array}\right) .
\end{aligned}
$$

Then $\varphi: S_{1} \longrightarrow S_{2}$ with

$$
\varphi\left(i_{1}, i_{2}\right)=i_{1}+2 \cdot i_{2}
$$

can be written as $\varphi:\left\langle S_{1}\right\rangle \longrightarrow\left\langle S_{2}\right\rangle$ with

$$
\begin{aligned}
& \varphi(\langle(a, a)\rangle)=\langle a\rangle+2 \cdot\langle a\rangle=3 \cdot\langle a\rangle=3 \cdot\left(\begin{array}{l}
1 \\
0
\end{array}\right)=\left(\begin{array}{l}
3 \\
0
\end{array}\right) \\
& \varphi(\langle(a, b)\rangle)=\langle a\rangle+2 \cdot\langle b\rangle=\left(\begin{array}{l}
1 \\
0
\end{array}\right)+2 \cdot\left(\begin{array}{l}
0 \\
1
\end{array}\right)=\left(\begin{array}{l}
1 \\
2
\end{array}\right) \\
& \varphi(\langle(b, a)\rangle)=\langle b\rangle+2 \cdot\langle a\rangle=\left(\begin{array}{l}
0 \\
1
\end{array}\right)+2 \cdot\left(\begin{array}{l}
1 \\
0
\end{array}\right)=\left(\begin{array}{l}
2 \\
1
\end{array}\right) \\
& \varphi(\langle(b, b)\rangle)=\langle b\rangle+2 \cdot\langle b\rangle=3 \cdot\langle b\rangle=3 \cdot\left(\begin{array}{l}
0 \\
1
\end{array}\right)=\left(\begin{array}{l}
0 \\
3
\end{array}\right) .
\end{aligned}
$$

Definition 10 The triple $\mathscr{N}=(P, T, F)$ is a petrinet, if the following holds:

$$
\begin{aligned}
P \quad: \quad & \text { is a set of places, with } P \neq \emptyset \\
& \text { and } \forall p \in P \text { a domain } D(p), \\
T: & \text { is a set of transitions, with } T \neq \emptyset \\
& \quad \text { and } \forall t \in T \text { a domain } D(t) \text { and } P \cap T=\emptyset, \\
F \quad \subseteq & (P \times T) \cup(T \times P) \text { is the flow relation. }
\end{aligned}
$$


We call $c_{p} \in D(p)\left(c_{t} \in D(t)\right)$ for $p \in P(t \in T)$ a place- or state-colour (transition- or firing-colour) and assume that $\forall n \in P \cup T|D(n)|<\infty$.

To avoid ambiguities we use in the context of nets the symbol \langle\rangle to represent the mapping of place-colours into vectors and the symbol [] to represent the mapping of transition-colours into vectors. So, with two linear spaces $V, W$ over the same field $K$ and an arbitrary transition-colour set $D(t)$ and a place colour set $D(p)$ with $\operatorname{dim}(V)=|D(t)|$ and $\operatorname{dim}(W)=|D(p)|$, there exists bijective mappings \langle\rangle and [ ] such that $\mathscr{L}(V, W)=\mathscr{L}(\mathbb{M S}([D(t)]), \mathbb{M S}(\langle D(p)\rangle))$ (see Figure 2).

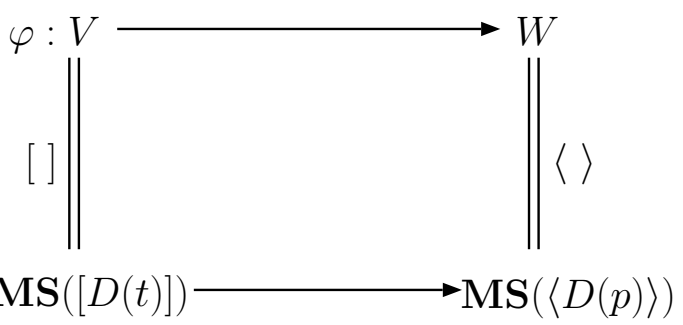

Figure 2: Vector-spaces and multisets of tuples.

Definition 11 Let $\mathscr{N}=(P, T, F)$ be a petrinet. For all $n \in P \cup T$ we define

$$
\begin{aligned}
& n:=\left\{n^{\prime} \mid\left(n^{\prime}, n\right) \in F\right\}-\text { the preset of } n \text { and } \\
& n \cdot:=\left\{n^{\prime} \mid\left(n, n^{\prime}\right) \in F\right\}-\text { the postset of } n .
\end{aligned}
$$

Definition 12 The quadrupel $\mathscr{N}=(P, T, F, L)$ is a predicate/transition-net (pr/t-net), if the following holds:

$$
\begin{aligned}
(P, T, F) \quad & \text { is a petrinet, } \\
L: \quad & F \longrightarrow\left\{\left\{\varphi, \varphi^{a d}\right\} \mid\right. \\
& \varphi \in \mathscr{L}(\mathbb{M} \mathbb{S}([D(t)]), \mathbb{M S}(\langle D(p)\rangle)), \\
& \varphi^{a d} \in \mathscr{L}(\mathbb{M} \mathbb{S}(\langle D(p)\rangle), \mathbb{M} \mathbb{S}([D(t)])), \\
& (p, t) \vee(t, p) \in F\} .
\end{aligned}
$$

That means: For all arcs $(t, p)$ and $(p, t)$ in a Pr/T-net there is a linear mapping $\varphi$ from $\langle\mathbb{M S}(t)\rangle$ to $\langle\mathbb{M S}(p)\rangle$ and in addition for the same arc a mapping $\varphi^{\text {ad }}$ from $\langle\mathbb{M S}(p)\rangle$ to $\langle\mathbb{M S}(t)\rangle$.

We often write $\varphi_{(a, b)}$ as an abbreviation for $\varphi \in \mathscr{L}((a, b))$ and $\varphi_{(a, b)}^{a d}$ for $\varphi^{a d} \in \mathscr{L}((a, b))$.

\section{SUCCESSIVE INFORMATION IMPROVEMENT IN PREDICATE/TRANSITION NETS}

Definition 13 Let $\mathscr{N}=(P, T, F, L)$ be a pr/t-net; the marking of $\mathscr{N}$ is defined as

$$
m: P \cup T \longrightarrow \bigcup_{p \in P, t \in T} \mathbb{M S}(\langle D(p)\rangle) \cup \mathbb{M S}([D(t)])
$$

with

$$
m(n) \in \begin{cases}\mathbb{M S}(\langle D(n)\rangle), & \text { if } n \in P \text { and } \\ \mathbb{M S}([D(n)]), & \text { if } n \in T\end{cases}
$$

The markings on transitions will be interpreted as sets of potential firing colours; the markings on places as sets of potential system states.

In the following the global marking of a net does corresponds to the knowledge about the state of the modeled system. Moreover, as we presume that the system's state is fixed, the dynamic in nets (defined below), and with it, the changing of markings, enables to change - here: to increase - the knowledge about the system's state.

Definition 14 Let $\mathscr{N}=(P, T, F, L)$ be a pr/t-net. The pre-place restriction of a transition $t \in T$ (in symbols: $r(\cdot t))$ is defined as

$$
r(\cdot t):=\bigcap_{p \in \cdot}\left(\bigcup_{c \in m(p)} \varphi_{(p, t)}^{a d}(c)\right) .
$$

The post-place restriction of a transition $t \in T$ (in symbols: $r(t \cdot))$ is defined as

$$
r(t \cdot):=\bigcap_{p \in t \cdot}\left(\bigcup_{c \in m(p)} \varphi_{(t, p)}^{a d}(c)\right) .
$$

Definition 15 Let $\mathscr{N}=(P, T, F, L)$ be a pr/t-net. The transition $t \in T$ is enabled under marking $m$ (in symbols $m[t\rangle)$, if

$$
m(t) \cap r(\cdot t) \cap r(t \cdot) \neq \emptyset .
$$

Definition 16 Let $\mathscr{N}=(P, T, F, L)$ be a pr/t-net. If $t \in T$ and $m[t\rangle$, then $t$ may fire; the firing of $t$ leads to the follower marking $m^{\prime}\left(m[t\rangle m^{\prime}\right)$ with

$$
\begin{aligned}
m^{\prime}(t) & =m(t) \cap r(\cdot t) \cap r(t \cdot), \text { and } \\
m^{\prime}(n) & =m(n) \forall n \neq t .
\end{aligned}
$$

Definition 17 Let $\mathscr{N}=(P, T, F, L)$ be a pr/t-net. The pre-transition restriction of a place $p \in P$ (in symbols: $r(\cdot p))$ is defined as

$$
r(\cdot p):=\bigcup_{t \in \cdot p}\left(\bigcup_{c \in m(t)} \varphi_{(t, p)}(c)\right) .
$$


The post-transition restriction of a place $p \in P$ (in symbols: $r(p \cdot))$ is defined as:

$$
r(p):=\bigcup_{t \in t^{\cdot}}\left(\bigcup_{c \in m(t)} \varphi_{(p, t)}(c)\right) .
$$

Definition 18 Let $\mathscr{N}=(P, T, F, L)$ be a pr/t-net. The place $p \in P$ is enabled under marking $m$ (in symbols $m[p\rangle)$, if

$$
m(p) \cap r(\cdot p) \cap r(p \cdot) \neq \emptyset .
$$

Definition 19 Let $\mathscr{N}=(P, T, F, L)$ be a pr/t-net. If $p \in P$ and $m[p\rangle$, then $p$ may fire; the firing of $p$ leads to the follower marking $m^{\prime}\left(m[p\rangle m^{\prime}\right)$ with

$$
\begin{aligned}
m^{\prime}(p) & =m(p) \cap(r(\cdot p) \cap r(p \cdot)), \text { and } \\
m^{\prime}(n) & =m(n) \forall n \neq p .
\end{aligned}
$$

For the pr/t-net in Figure 3 the following holds:

$$
\begin{aligned}
& P=\{P 1, P 2\}, \text { with } D(P 1)=D(P 2)=\{0 ; \ldots ; 1\} \\
& T=\{T 1\}, \text { with } D(T 1)=\{0 ; \ldots ; 1\} \times\{0 ; \ldots ; 1\} \\
& F=\{(P 1, T 1),(T 1, P 2)\}
\end{aligned}
$$

with the arc-lables being specified as follows:

$$
\begin{gathered}
L((P 1, T 1))=\left\{\varphi_{(P 1, T 1)}, \varphi_{(P 1, T 1)}^{a d}\right\}: \\
\varphi_{(P 1, T 1)}: \mathbb{M S}([D(T 1)]) \longrightarrow \mathbb{M S}(\langle D(P 1)\rangle), \text { with } \\
\varphi_{(P 1, T 1)}\left(\left[p_{1}, x\right]\right)=\left\langle p_{1}\right\rangle \text { and } \\
\varphi_{(P 1, T 1)}^{a d}: \mathbb{M} \mathbb{S}(\langle D(P 1)\rangle) \longrightarrow \mathbb{M S}([D(T 1)]), \text { with } \\
\varphi_{(P 1, T 1)}^{a d}\left(\left\langle p_{1}\right\rangle\right)=\sum_{x}\left[p_{1}, x\right] .
\end{gathered}
$$

and

$$
L((T 1, P 2))=\left\{\varphi_{(T 1, P 2)}, \varphi^{a d}{ }_{(T 1, P 2)}\right\}:
$$

$\varphi_{(T 1, P 2)}: \mathbb{M S}([D(T 1)]) \longrightarrow \mathbb{M S}(\langle D(P 2)\rangle)$, with

$\varphi_{(T 1, P 2)}\left(\left[p_{1}, x\right]\right)=\left\langle p_{1}-\left(p_{1}\right) \cdot x\right\rangle$ and

$\varphi^{a d}{ }_{(T 1, P 2)}: \mathbb{M S}(\langle D(P 2)\rangle) \longrightarrow \mathbb{M S}([D(T 1)])$, with

$\varphi_{(T 1, P 2)}^{a d}\left(\left\langle p_{2}\right\rangle\right)=\sum_{p_{1}}\left[p 1, \frac{p_{1}-p_{2}}{p_{1}-1}\right]+\left(\left\langle p_{2}\right\rangle \bullet\langle 1\rangle\right) \cdot \sum_{x}[1, x]$.

Here the set $\{0 ; \ldots ; 1\}$ specifies a set of values in the interval $[0, \ldots, 1]$ and (multi-)sets are again written as sums.

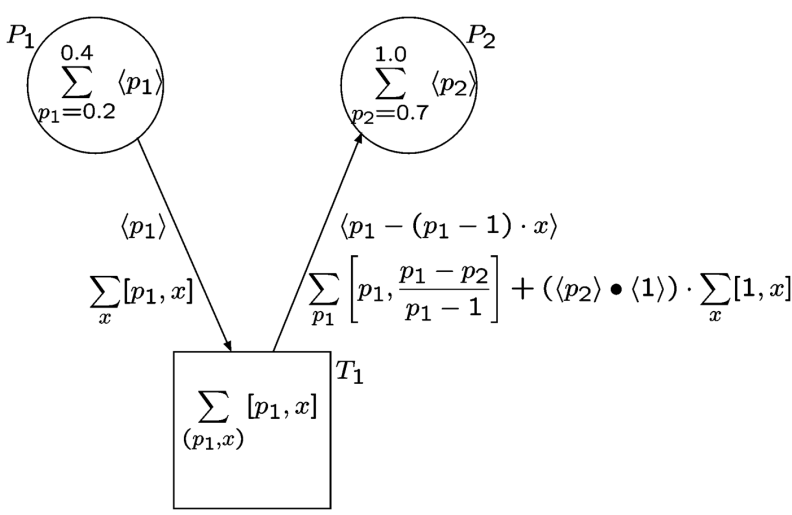

Figure 3: Abstract pr/t-net with initial marking.

The initial marking $m_{0}$ of $\mathscr{N}$ is given as follows:

$$
\begin{aligned}
& m_{0}(P 1)=\sum_{p_{1}=0.2}^{0.4}\left\langle p_{1}\right\rangle \\
& m_{0}(T 1)=\sum_{\left(p_{1}, x\right) \in D(T 1)}\left[p_{1}, x\right] \\
& m_{0}(P 2)=\sum_{p_{2}=0.7}^{1.0}\left\langle p_{2}\right\rangle .
\end{aligned}
$$

The initial marking can be interpreted as follows: The local state of $P 1$ is a value in the interval 0.2 to 0.4 ; furthermore it is known, that the local state of $P 2$ lies somewhere between 0.7 and 1.0. The local marking on transition $T 1$ is the most unspecified one: we only know the potential firing colours $c$ of $T 1$ are in $D(T 1)$.

To narrow down the marking on $T 1$ and, the marking on $T 1$ is restricted in the defined manner: Therefore it's restrictions $r(\cdot T 1)$ and $r(T 1 \cdot)$ have to be calculated:

$$
\begin{aligned}
r(\cdot T 1) & =\bigcup_{p_{1} \in\{0.2, \ldots, 0.4\}} \varphi^{a d}{ }_{(P 1, T 1)}\left(\left\langle p_{1}\right\rangle\right) \\
& =\bigcup_{p_{1} \in\{0.2, \ldots, 0.4\}} \sum_{x}\left[p_{1}, x\right]
\end{aligned}
$$

(see Figure 4 (left) for grahical representation of $r(\cdot T 1)$ ).

$$
\begin{array}{r}
r(T 1 \cdot)=\bigcup_{p_{2} \in\{0.7, \ldots, 1.0\}} \varphi^{a d}{ }_{(T 1, P 2)}\left(\left\langle p_{2}\right\rangle\right) \\
=\bigcup_{p_{2} \in\{0.7, \ldots, 1.0\}} \sum_{p_{1}}\left[p_{1}, \frac{p_{1}-p_{2}}{p_{1}-1}\right]+ \\
\left(\left\langle p_{2}\right\rangle \bullet\langle 1\rangle\right) \cdot \sum_{x}[1, x]
\end{array}
$$

(see Figure 4 (right) for grahical representation of $r(T 1 \cdot)$ ). 


\section{Müller and Schnieder}
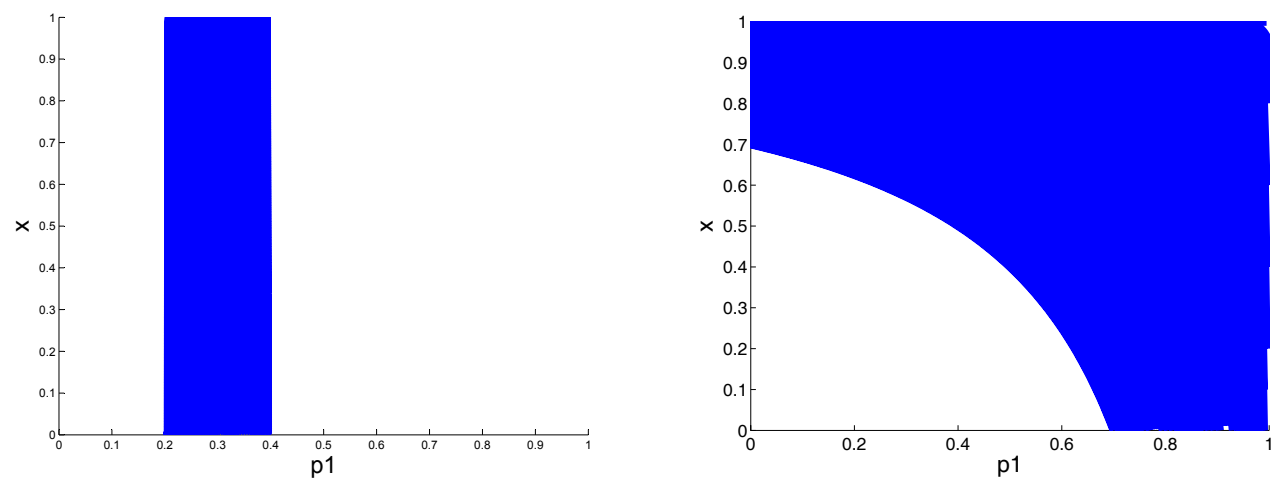

Figure 4: Restrictions onto $T 1: r(\cdot T 1)$ (left) and $r(T 1 \cdot)$ (right). ure 4)

So, with $m(T 1)=\sum_{(p 1, x)}\left[p_{1}, x\right]$ we get here (see Fig-

$$
\begin{aligned}
m^{\prime}(T 1) & =m(T 1) \cap r(\cdot T 1) \cap r(T 1 \cdot) \\
& =r(\cdot T 1) .
\end{aligned}
$$

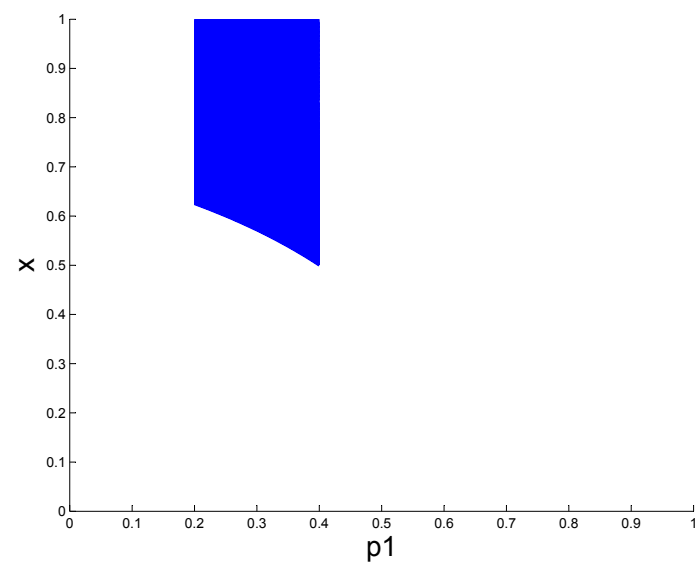

Figure 5: Graphical representation of the modified marking on $T 1$.

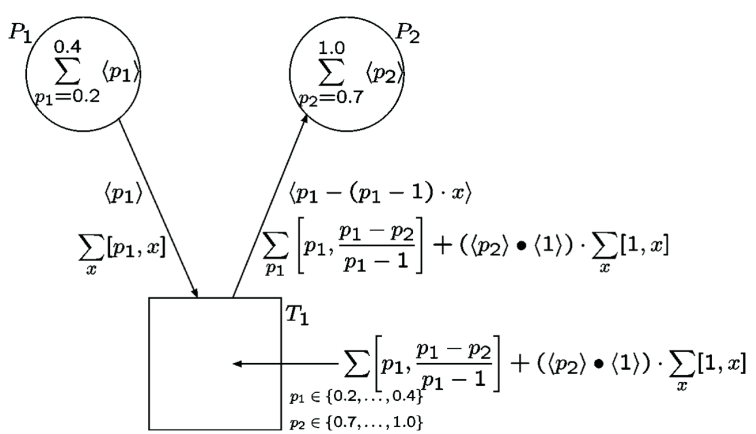

Figure 6: Pr/t-net with modified marking on $T 1$.

\section{EXAMPLE}

In this section the concept of duality in high-level nets is applied in an example from the automotive area. On a simplified airpath of a turbo engine system (see Figure 7) the above described process of successive information improvement due to restrictions leads to a formal diagnosis method. Please have in mind that it is just and example to demonstrate a possible application of the intorduced approach in reality the system is more complex.

The airpath under consideration consists of a throttle valve that, in dependence of the accelerator's pedal position, influences the amount of incoming fresh air (full acceleration: throttle valve is fully opened; idle - no acceleration: throttel valve is (nearly) closed). The pressure of the air flow between the throttle valve and the compressor is called "map_up"-value ("Magnifold Absolute Pressure Upstream"). Due to the compressor the air is (at least in general) compressed and taken into the engine; the pressure in the air hose between the compressor and the engine intake is called the "map"-value ("Magnifold Absolute Pressure"). Since during full acceleration the throttle valve is fully opened and the bypass-valve is closed, a pressuredifference (map-map_up) arises; during idling the throttle valve is nearly closed and the bypass-valve is fully opened so, map = map_up (pressure balance due to opened bypassvalve). In our scenario we presume that the position of the throttle valve, the map_up- als well as the map-value can be established as faultless - so, we can rely on these values. As potential sources of errors, we consider:

- a leak between throttle valve and compressor (modelled by transition Leak 1 in our model - see Figure 8; the leak's size is modelled by the value of variable $l 1$,

- a leak between the compressor and the engine (in the model: transition Leak2 with size of value 12 ) and

- a potentially faulty bypass-valve (transition bypass - valve, value: by).

Assume now, that at the working point "full acceleration" $(d k=1)$ the measured pressure values are mapup $=0.7$ and map $=1.18-$ in contrary to the expected reference values: mapup $=0.5$ and map $=0.7$ (with $l_{1}=l_{2}=0$ and $b y=1)$. A diagnosis applying the method described 


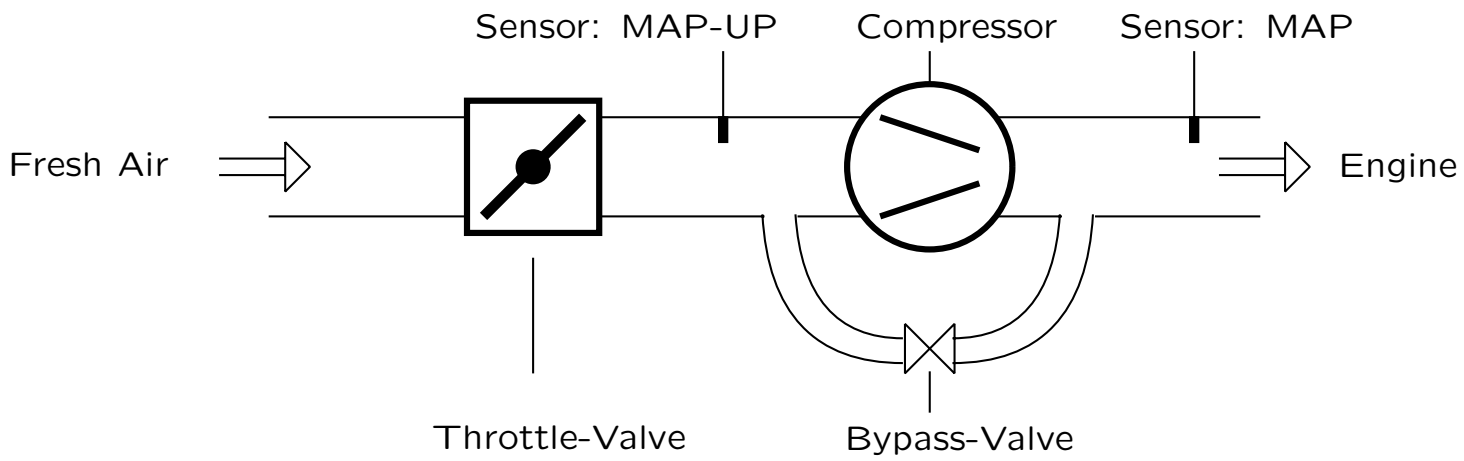

Figure 7: The schema of a simplified airpath.

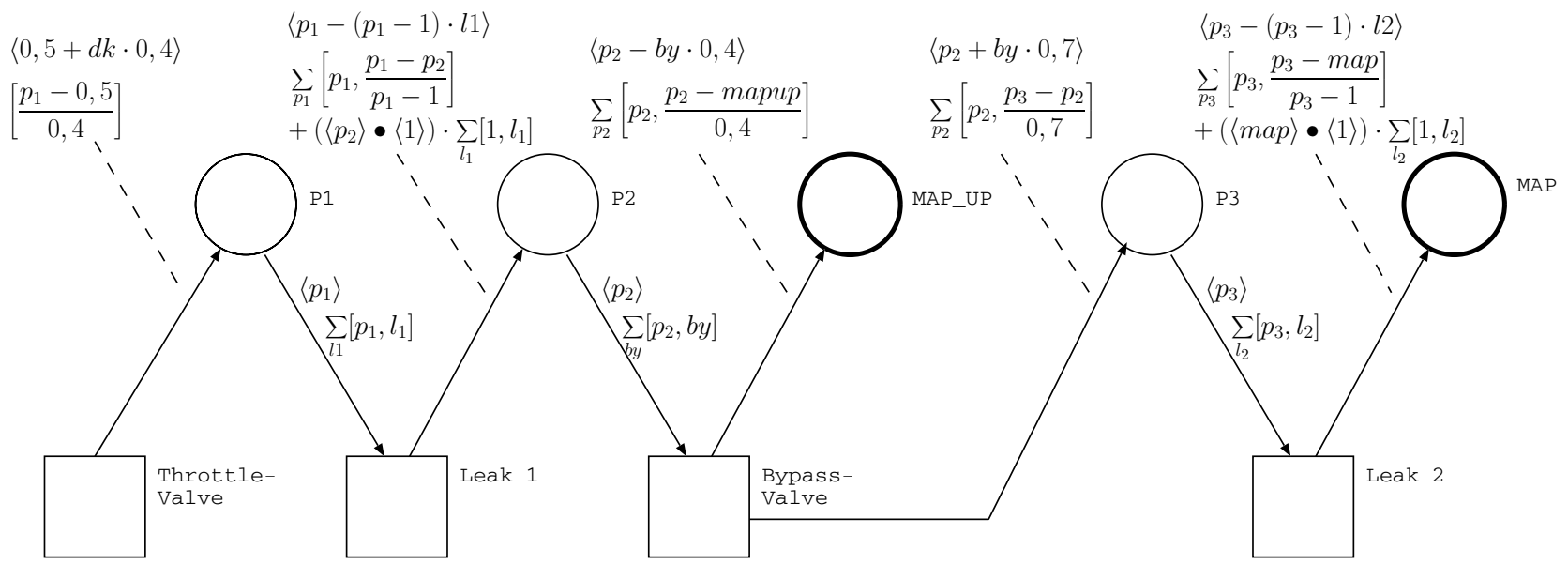

Figure 8: The model of a simplified airpath.

in chapter 3 on the basis of the measured values (and the markings on all nodes $n \in P \cup T \backslash\left\{\right.$ mapup, map,$\left.p_{1}\right\}$ set to $\langle D(n)\rangle$ and $[D(n)]$ respectively), leads to the faultspace shown in Figure 9 (left) - each point of the surface constitutes a potential (combination of) fault(s) consisting of a leak1, leak2 and a faulty bypass-valve. With the second fault-space - generated in dependence of the second working point "idly - no acceleration" (reference values: mapup $=0.5$, map $=0.5$; measured values: mapup $=0.3$, map $=0.94$ ) and depicted together with the first fault-space in Figure 9 (right) - one can by intersecting both spaces derive the combination of faults leading to the observed values: $l_{1}=0, b y=0.5$ and $l_{2}=0.4$. That means: there's no leak between the throttle valve and the compressor, the bypass-valve sticks half-opened and there's a leak of size 0.4 between the compressor and the engine.

\section{CONCLUSION AND FUTURE WORK}

In this paper we have defined a class of high level Petri-nets on the basis of the theory of dual spaces. In that class places as well as transitions can be marked and each arc carries two labels - the primal mapping and the corresponding adjoint mapping. If we assume that a system's state is only known with uncertainty and not exactly the interpretation of tupels as "the states that can't be excluded" suggests itself. With the help of the two mappings on each arc and the markings on places and transitions it was shown, that the knowledge about the system's state can (at least in general) be improved by passing the (uncertain) information through the model and checking it for consistence.

The next tasks that must be dealt with are to examine how far dual/adjoint mappings do exist (and can be generated) to non-linear mappings (see arc labels (Leak1,P2) and (Leak2,P3) of example in Figure 8). Up to now, it has only been proved that adjoint mappings do exist for linear mappings of finite dimension but we could find adjoints to many non-linear mappings in different applications.

Secondly, the combination with "module-nets" (see Simon 2005) is under consideration for model-refinement tasks: In the approach presented here, we presume that the arc labels are fixed and that the (observed) markings are varyable. We could imagine that presuming fixed markings but "bendy" arc labels is an approach that is worth to think about. 

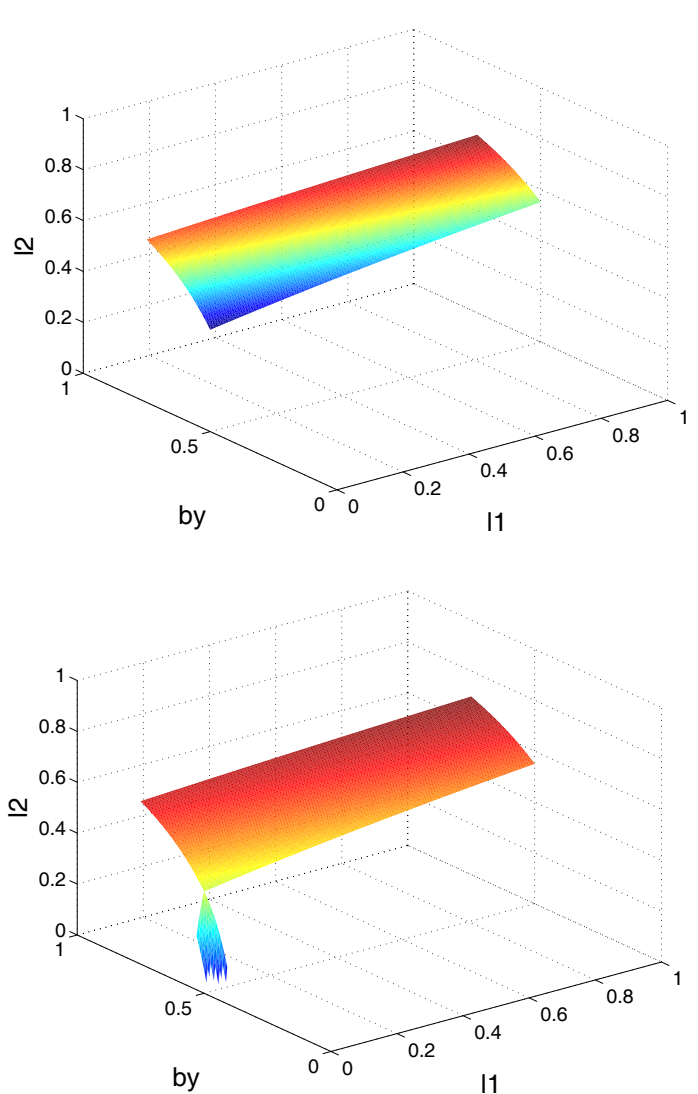

Figure 9: Fault spaces at working point 1 (upper part) and working point $1+2$ (lower part).

\section{REFERENCES}

Blyth, T. S., and E. F. Robertson. 2002. Further Linear Algebra. London, Berlin, Heidelberg: Springer.

Curtis, M. L. 1990. Abstract Linear Algebra. New York, Berlin, Heidelberg: Springer.

Fernandez, C. 1975. Net Topology I. Bonn: Gesellschaft fuer Mathematik und Datenverarbeitung mbH Bonn, Interner Bericht ISF-75-09, September.

Fischer, G. 2002. Lineare Algebra - Eine Einführung für Studienanfänger, 13. auflage. Braunschweig, Wiesbaden: Vieweg.

Genrich, H. J., and K. Lautenbach. 1979. The Analysis of Distributed Systems by Means of Predicate-/Transition Nets. In $\operatorname{LNCS} 70,123$ - 146.

Gilbert, J., and L. Gilbert. 1994. Linear Algebra and Matrix Theory. San Diego, New York, Boston: Academic Press.

Kowalsky, H.-J. 1979. Lineare Algebra, 9. Auflage. Berlin, New York: de Gruyter Lehrbuch.

Kruse, R. J. 2001. Dualität bei Petri-Netzen - Anwendungen fuer Netze mit Stellen- und Transitionsmarken. Koblenz: Koblenzer Schriften zur Informatik, Foelbach.
Lautenbach, K. Juli 1983. Simple Marked-graph-like Predicate/Transition Nets. Bonn: Arbeitspapiere der GMD Nr. 41, Informatik Fachberichte 66.

Lautenbach, K., and A. Pagnoni. 1984. Liveness and Duality in Marked-graph-like Predicate/Transition Nets. Berlin, Heidelberg, New York, Tokyo: Springer-Verlag.

Lautenbach, K., and A. Pagnoni. 1985. Invariance and Duality in Predicate/Transition Nets and in Coloured Nets. Bonn: Gesellschaft fuer Mathematik und Datenverarbeitung $\mathrm{mbH}$.

Lipschutz, W., and M. Lipson. 2001. Schaum's outlines Linear Algebra (Third Edition). New York, London, Madrid: Mc Graw-Hill.

Müller, J. R. 2004. Dualitt und Analyse von Formalen Modellen - Prdikat/Transitions-Netze und ihr Bezug zur Linearen Algebra. Koblenz: Foelbach - Koblenzer Schriften zur Informatik.

Murata, T. 1989. Petri nets: properties, analysis and application. In Proceedings of the IEEE, Vol 77, 541 580.

Simon, C. 2005.. Incremental Development of Business Process Models. In Development Methods for Information Systems and their Application., ed. J. U. Frank.

Smith, E. 1998. Principles of High-Level Net Theory. Lecture Notes in Computer Science: Lectures on Petri Nets I: Basic Models 1491:174-210.

\section{AUTHOR BIOGRAPHIES}

JÖRG R. MÜLLER is a Research Assistant at the Institute for Traffic Safety and Automation Engineering (Technical University of Braunschweig, Germany). His research interests are in formal models and analyses, duality and diagnoses, and safety and reliability in the automotive and railway domain.

ECKEHARD SCHNIEDER is a Professor at the Technichal University of Braunschweig, and is Director of the Institute for Traffic Safety and Automation Engineering. 